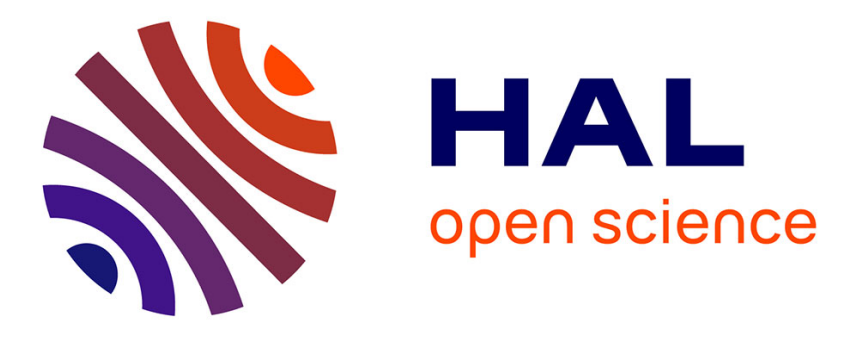

\title{
Development of an optically accessible apparatus to characterize the evolution of spherically expanding flames under constant volume conditions
}

Fabien Halter, Zheng Chen, Guillaume Dayma, Chaimae Bariki, Yong Wang, Philippe Dagaut, Christian Chauveau

\section{To cite this version:}

Fabien Halter, Zheng Chen, Guillaume Dayma, Chaimae Bariki, Yong Wang, et al.. Development of an optically accessible apparatus to characterize the evolution of spherically expanding flames under constant volume conditions. Combustion and Flame, 2020, 212, pp.165-176. 10.1016/j.combustflame.2019.10.027 . hal-02357258

\section{HAL Id: hal-02357258 \\ https://hal.science/hal-02357258}

Submitted on 13 Oct 2020

HAL is a multi-disciplinary open access archive for the deposit and dissemination of scientific research documents, whether they are published or not. The documents may come from teaching and research institutions in France or abroad, or from public or private research centers.
L'archive ouverte pluridisciplinaire HAL, est destinée au dépôt et à la diffusion de documents scientifiques de niveau recherche, publiés ou non, émanant des établissements d'enseignement et de recherche français ou étrangers, des laboratoires publics ou privés. 


\title{
Development of an optically accessible apparatus to characterize the evolution of spherically expanding flames under constant volume conditions
}

\author{
F. Halter ${ }^{* 1,2}$, Z. Chen ${ }^{3}$, G. Dayma ${ }^{1,2}$, C. Bariki ${ }^{1,2}$, Y. Wang ${ }^{3}$, P. Dagaut ${ }^{1}$, C. Chauveau ${ }^{1}$ \\ ${ }^{1}$ CNRS ICARE, Avenue de la Recherche Scientifique, 45071 Orléans Cedex 2 France \\ ${ }^{2}$ Université d’Orléans, Orléans, France \\ ${ }^{3}$ SKLTCS, College of Engineering, Peking University, Beijing, China
}

*Corresponding author: fabien.halter@cnrs-orleans.fr

\begin{abstract}
:
Flame speed is extremely important as it affects the performances of many industrial systems. Moreover, its significance makes it a major target for the validation of kinetic mechanisms, which explains the necessity to provide ever more accurate data. Flame speed dependence on pressure and temperature conditions is interestingly assessed using, among others, spherically expanding flame in constant volume chambers. In these conditions, the flame speed derivation, based solely on the pressure evolution in the chamber, requires empirical models. The current study describes a perfectly spherical chamber with full optical access allowing simultaneous recording of the pressure inside the chamber and, fully innovative, of the flame radius evolution until the flame vanishes at wall. A careful description of the new set-up and of the accuracy of the measurements, in particular of the flame radius, are presented here. In parallel with experiments, one-dimensional transient simulations were carried out to identify the limits of the proposed new method. Then, the simultaneous use of pressure and flame radius information is compared to the traditional constant volume method based on empirical models. A first advantage relies in the direct detection of the development of instabilities during the flame propagation. In addition, although the flame speed is extremely sensitive to the flame radius determination, the actual experimental accuracy allows significant improvements in terms of accuracy, notably as initial pressure and temperature are elevated. This new set-up will allow major advances in the measurement of laminar flame velocity under extreme thermodynamic conditions.
\end{abstract}

Keywords: Laminar flame speed; Uncertainty quantification; Engine conditions; Spherically expanding flame; Constant volume chamber 


\section{Introduction}

The laminar flame speed is defined as the propagation speed relative to the unburnt mixture of a steady, laminar, one-dimensional, planar, stretch-free, and adiabatic flame, hereafter referred to as freely propagating flame. It is one of the most important parameters of a combustible mixture. On a practical level, it affects the fuel burning rate in internal combustion engines and the stabilisation process and engine performance of gas turbines [1]. On a more fundamental level, it is an important target for the validation of kinetic mechanisms [2]. Accurate determination of flame speeds at high pressures and temperatures is extremely important for the development of kinetic mechanisms and ensure their validity in the simulation of industrial configurations.

Several methods have been developed to experimentally measure the laminar flame speed [3]. One of the most common methods is to follow the evolution of a spherical flame propagating outward in a confined bomb. This method allows the measurement of laminar flame speeds at relatively high initial pressure and temperature conditions. Indeed, it allows exceeding 10 bar, which is difficult for other methods using stationary flames such as Bunsen flame, counterflow flame or burner stabilised flat flame. The reasons of these pressure $(P)$ and unburnt temperature $(T)$ limitations were clearly identified by Xiouris et al. [4]. The limitation in temperature is almost the same for all the systems. Sealing of the optical accesses is one of the key issues. This is avoided by using externally heated system [5] or shock heating [6]. Pressure limitation is closely linked to the mechanical resistance of the systems but also because of the onset of instabilities on the flame surface. One solution to delay the onset of flame instabilities is to replace air with a mixture of helium and oxygen as done by Tse et al. [7].

Jayachandran et al. [8] pointed that most of the flame studies have been conducted either at quasisteady thermodynamic conditions or under unsteady pressure rise conditions (i.e. isobaric spherically expanding flames, SEFs) but interpreted based on the assumption that pressure is quasi-constant [9, 10]. From a very pragmatic point of view, each measurement performed using isobaric SEF method or stationary systems allows to obtain a unique laminar flame speed for the given conditions. Acquiring a complete database is thus time and money consuming. In addition, an indirect consequence is that, because of the relative simplicity of performing experiments at ambient conditions, most of the data available are for these conditions. As a result, the weight given for kinetics schemes validation to the data obtained at ambient conditions is far too important considering the industrial purposes (i.e. engine and gas turbines). Indeed, Xiouris et al. [4] pointed out that, based on a survey of nearly 120 papers published in major combustion journals on the experimental determination of laminar burning velocity, only $27 \%$ were obtained for $P>1$ bar with a drastic decrease of this number for $P>$ 10 bar.

The constant volume method proposed by Lewis and von Elbe [11] uses the unsteady pressure-time history during the flame propagation event. Conversion of the reactants to hot products across the flame front results in a rapid pressure rise and a corresponding temperature rise in unburnt and burnt gas. The constant volume technique seeks to relate the instantaneous flame speed to the pressure history. In other words, in a single test, flame speeds can be obtained for a range of higher pressures and temperatures [10]. Recently, Hinton et al. [12] pointed out the fact that the method using the pressure rise requires a more complex analysis, but has the advantage that a single experiment generates data across a range of linked temperatures and pressures [13]. The pressure and temperature rise also means that data can be obtained for engine-like conditions [2].

Equations for this method has been the subject of several pioneered publications [14, 15]. Based on the following assumptions: the pressure $(P)$ is spatially uniform; the constituents of the burnt and unburnt gas behave as ideal gases, the following expression for the flame speed $\left(S_{u}\right)$ was derived [16]: 
$S_{u}=\frac{d R_{f}}{d t}-\frac{\left(R_{0}^{3}-R_{f}^{3}\right)}{3 R_{f}^{2}} \frac{1}{\rho_{u}} \frac{d \rho_{u}}{d t}$

where $R_{f}$ and $R_{0}$ stand for the flame radius and the inner chamber radius, respectively and $\rho_{u}$ corresponds to the unburnt gas density.

Experimental evaluation of the compression term $\left(\frac{1}{\rho_{u}} \frac{d \rho_{u}}{d t}\right)$ is possible but delicate [17]. Alternatively, assuming that the unburnt gas is isentropically compressed (with $\gamma_{u}$ the heat capacity ratio of the unburnt gas) yields:

$S_{u}=\frac{d R_{f}}{d t}-\frac{\left(R_{0}^{3}-R_{f}^{3}\right)}{3 \gamma_{u} R_{f}^{2} P} \frac{d P}{d t}$

To evaluate the flame speed, both the pressure and the flame radius need to be determined simultaneously over a sufficiently long period of time to enable the computation of fairly accurate derivatives. To overcome the lack of optical access in conventional isobaric combustion chambers, a relation linking the flame radius to the pressure is usually used:

$\frac{R_{f}}{R_{0}}=\left[1-(1-x)\left(\frac{P_{0}}{P}\right)^{1 / \gamma_{u}}\right]^{1 / 3}$

with $P_{0}$ the initial pressure in the chamber and $x=\frac{m_{b}}{m}$ the burnt gas mass fraction (with $m$ the total mass and $m_{b}$ the burnt gas mass). Similar to the flame radius, the burnt gas mass fraction is not accessible experimentally. The $x=f(P)$ relation is either determined via numerical modelling $[15,18$, 19] or via simplified assumptions. The simple linear $x-P$ relation initially derived by Lewis and Von Elbe [11] is still widely used:

$x=\frac{P-P_{0}}{P_{e}-P_{0}}$

with $P_{e}$ the theoretical end-pressure, which should be evaluated using thermo-chemical calculations. Its value significantly affects the flame speed determination and may be influenced by dissociation [20].

The validity of this relation, which violates energy conservation, was tested earlier [21, 22]. A more accurate $x-P$ relation that takes into account the temperature rise in both the burnt gas zone and the unburnt gas zone was proposed by Luijten et al. [23]:

$x=\frac{P-P_{0} \cdot f(P)}{P_{e}-P_{0} \cdot f(P)}$

with $f(P)=\frac{\gamma_{b}-1}{\gamma_{u}-1}+\frac{\gamma_{u}-\gamma_{b}}{\gamma_{u}-1}\left(\frac{P}{P_{0}}\right)^{\left(\gamma_{u}-1\right) / \gamma_{u}}$

Both unburnt $\left(\gamma_{u}\right)$ and burnt $\left(\gamma_{b}\right)$ heat capacity ratios are used in the above equation. The burnt mass fraction, $x$, was found very sensitive to the value of $\gamma_{b}$ [9]. Considering a stoichiometric $\mathrm{CH}_{4} / \mathrm{Air}$ at normal pressure and temperature conditions, $\gamma_{b}$ changes from 1.27 to 1.25 during the flame propagation. However, chemical equilibrium shifting and radiation in burnt gas substantially decrease the value of $\gamma_{b}$. Faghih and Chen [9] proposed an adjusted value: $\gamma_{b_{-} \text {Faghih }}=\frac{8+\gamma_{b}}{8}$.

Assuming an isentropic compression of the unburnt gas due to the burnt gas expansion, the $P-T$ trace may be estimated from the initial pressure and temperature conditions (with $T$ the unburnt temperature). If no heat losses occur between the unburnt compressed gas and the chamber wall, the final chamber pressure reaches the theoretical end-pressure, which is never the case. The upper limit 
in the flame radius must guarantee no heat losses to the wall [25]. The maximal radius considered by Yamamoto et al. [26] is $99 \%$ of the total chamber dimension. Considering the strict limitations fixed by Omari et al. [22] (i.e. 55\% of the peak pressure), we can sketch a map of the conditions achievable with the constant volume methodology as presented in Figure 1. In a first-order approximation, a value of $\gamma_{u}=1.4$ was considered. We also assume that instabilities may be avoided using helium as done in [27-29]. To be consistent with experimental limitations, the maximal initial conditions were set to 10 bar and $200{ }^{\circ} \mathrm{C}$. The conditions achievable by other methods (i.e. constant pressure chamber [7, 30], heat flux burner $[31,32]$, Bunsen burner [33, 34], externally heated channel $[35,36]$, stagnation/counter-flow burners $[37,38]$ ) are also indicated in Figure 1.

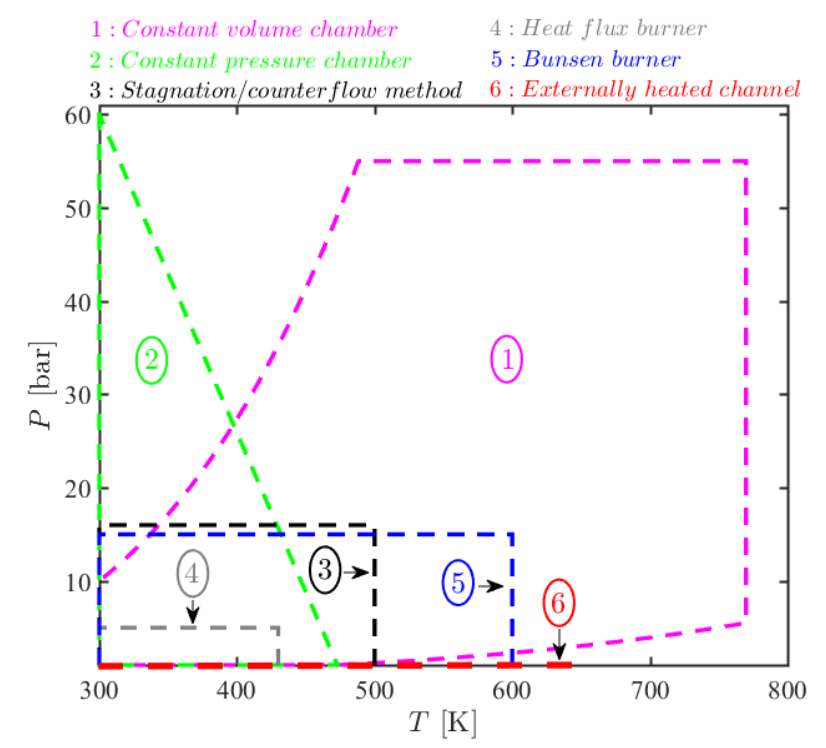

Figure 1 : Map of $P \& T$ conditions reachable when maximal initial conditions are limited to 10 bars and $200{ }^{\circ} \mathrm{C}$. Areas of use of other experimental devices are also reported.

The enormous potential of the isochoric method jumps out and clearly justifies the interest that the combustion community has been carrying for decades. However, Jayachandran et al. $[4,8]$ have pointed out several sources of uncertainty: flame area growth due to cellular instability, influence of flame stretch and radiation, accuracy of the $R_{f}(P)$ model and effect of transient pressure rise.

Aware of the numerous advantages offered by the isochoric SEF method, our objective was to develop a new experimental device pushing further its present limitations. A brand-new perfectly spherical isochoric combustion chamber with full optical access (OPTIPRIME) was designed in order to benefit from the great potential of this method. 


\section{Experimental set up description}

\subsection{The chamber and its equipment}

\subsubsection{A perfectly spherical combustion chamber with full optical accesses}

In the specifications of this new chamber, two conditions needed to be strictly fulfilled: a perfect sphericity of the inner chamber surface and a full visualisation of the flame front propagation from the central ignition to the walls. The use of a $360^{\circ}$ fused silica ring for the flame visualisation was the selected solution. Inner and outer surfaces of this fused silica ring are machined to ensure perfect internal and external spherical profiles.

The internal diameter of the ring, identical to the chamber inner diameter, guarantees a perfect sphericity inside the chamber. The outer diameter of the crown is $144 \mathrm{~mm}$ corresponding to a quartz thickness of $11 \mathrm{~mm}$. This thickness was calculated to hold at pressure greater than 100 bars.

OPTIPRIME consists of the assembly of two stainless steel half-shells with a $360^{\circ}$ fused silica ring with spherical inner and outer surfaces in-between as illustrated in Figure 2.

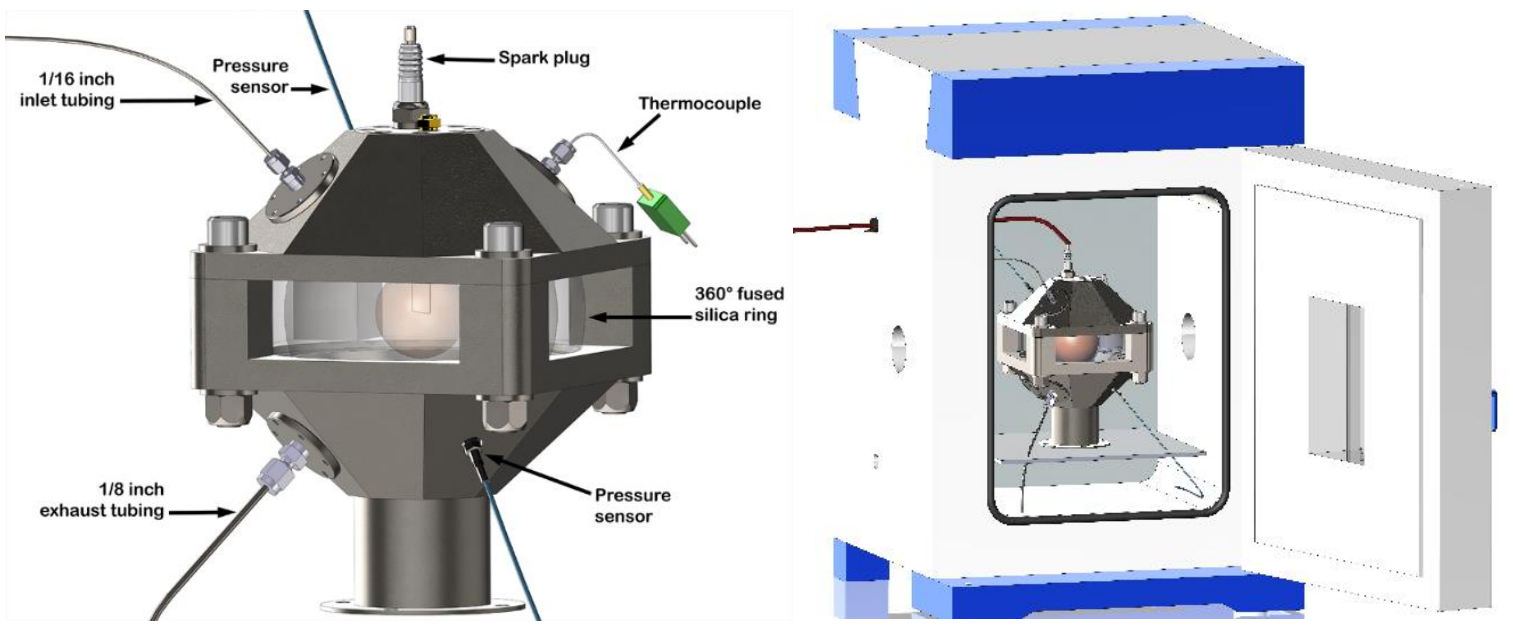

Figure 2 : Sketch of the spherical chamber (left) and of the chamber in the furnace (right)

The internal volume is scrupulously spherical, and its radius is $R_{0}=60.65 \mathrm{~mm}$ (i.e. 0.934 liter in volume). There are several important advantages to smaller combustion bombs. First, it is much easier to heat up in order to get a homogeneous pre-mixture. Moreover, the effects of radiation and hydrodynamic instabilities are less pronounced at small flame radii. One drawback of working with small flames is that they are more strongly affected by stretch.

To minimise dead volumes, the diameters of the inlet (1/16 inch) and exhaust (1/8 inch) tubing were reduced and their end is flush with the inner surface of the chamber. The exhaust tubing is larger to facilitate the vacuum procedure after each test.

Two pressure sensors (AVL GU21D), with high sensitivity and high frequency, are arranged diametrically opposite in the chamber. The sensitive surface of the sensors is flush with the wall. The sensitivity and frequency of the sensor are $35 \mathrm{pC} /$ bar and $85 \mathrm{kHz}$ respectively. The linearity of the sensor is $\pm 0.3 \%$ of the full-scale range.

A type-K thermocouple, stainless steel sheathed, is introduced into the chamber to control the initial temperature of the unburnt gas. As shown in Figure 2, the combustion chamber is placed in the centre 
of a furnace with a volume of 32 litres, a power of $1050 \mathrm{~W}$ and capable of operating over a temperature range of $10^{\circ} \mathrm{C}$ to $300^{\circ} \mathrm{C}$ with a time stability lower than $0.2^{\circ} \mathrm{C}$. A complete immersion of the chamber has the major advantage of avoiding temperature gradients either on the enclosure or in unburnt gas.

OPTIPRIME was designed to hold a maximum pressure of 100 bar. The maximum initial temperature $\left(200^{\circ} \mathrm{C}\right)$ is actually limited by the thermal resistance of the pressure sensor cables, as the whole system is in the furnace.

\subsubsection{Mixture preparation and ignition}

Air and fuel are mixed prior to their introduction into the chamber, using a high-pressure buffer tank (3.78 litres). Fuels, oxidizer and diluents are successively introduced in the tank following Dalton's law of partial pressures, and measuring the static pressure and temperature at each step via pressure transducers and a type-K thermocouple. The pressure transducers used are KELLER LEO 2. For such transducers the uncertainty in the pressure is $0.1 \%$ of the full range of the device. Thus, in order to improve accuracy, we use two transducers with different ranges and operate as close to full scale as possible. The relatively large volume of the buffer tank allows carrying out several successive shots tests with exactly the same mixture. Note that the mixture is sampled to verify its composition by gas chromatography. Before each filling, the chamber is flushed with air. Then, it is vacuumed and filled with the flammable mixture until the desired initial pressure.

The ignition of the mixture is ensured by an electric arc generated by two ultra-fine electrodes, 0.35 $\mathrm{mm}$ in diameter, arranged face to face as illustrated in Figure 2. The inter-electrode distance is set to $1.5 \mathrm{~mm}$. The electrodes need to be perfectly positioned in the centre of the chamber. The reproducibility and reliability of the method require the greatest care to ensure perfect centring of the ignition device, which is essential to obtain that the flame in all directions reaches the chamber walls at the same time. The ignition system is mounted on a removable flange, allowing precise assembly and adjustment through the use of an adjustment gauge. The energy deposit is controlled by the charging time of a capacitive coil. A constant value of this energy has been maintained throughout the experiments presented here, and maintained close to the minimum ignition energy.

The experimental rig was designed to be run with heavy fuels at high pressure and temperature conditions. However, because of the novelty in the set-up, a validation step with a well-known fuel seemed essential to us. Thus, for the sake of simplicity, methane/air mixtures were tested in this study. Two equivalence ratios were selected: a stoichiometric $(\phi=1.0)$ and a fuel-rich $(\phi=1.3)$ conditions. The fuel-rich mixture was selected to limit the occurrence of cellularity at high-pressure conditions. Two initial conditions were selected as indicated in Table 1: an atmospheric condition $\left(T_{0}=300 \mathrm{~K} \& P_{0}=1 \mathrm{bar}\right)$ and a higher $P \& T$ condition $\left(T_{0}=404 \mathrm{~K} \& P_{0}=3 \mathrm{bar}\right)$. The latter corresponds to a compromise to obtain elevated pressure and temperature conditions during the flame propagation while ensuring the stability of the rich mixture condition. Theoretical end pressures considering an adiabatic and isochoric combustion were evaluated using the CHEMKIN equilibrium calculator and are reported in Table 1.

Table 1 : Initial mixture conditions tested.

\begin{tabular}{lccccc}
\hline Fuel & Oxidizer & $T_{0}(\mathrm{~K})$ & $P_{0}($ bar $)$ & $\phi(-)$ & $P_{e}$ (bar) \\
\hline $\mathrm{CH}_{4}$ & Air & 300 & 1 & 1 & 8.72 \\
$\mathrm{CH}_{4}$ & Air & 404 & 3 & 1 & 19.97 \\
\hline $\mathrm{CH}_{4}$ & Air & 300 & 1 & 1.3 & 8.73 \\
$\mathrm{CH}_{4}$ & Air & 404 & 3 & 1.3 & 19.98 \\
\hline
\end{tabular}


The end gas pressures of the two mixtures $(\phi=1.0 \& \phi=1.3)$ are almost equivalent for each initial condition. For the 3 bar condition, $P \& T$ reached by the unburnt gas at the end of the compression will exceed 15 bar and $600 \mathrm{~K}$.

In addition to the intrinsic accuracy of each sensor, the initial conditions can be slightly different from one experiment to another. We have defined tolerance criteria that can be modified according to the level of precision required. For pressure and temperature, we have allowed a maximal variation of $+/$ $1.5 \mathrm{~K}$ and $+/-0.01$ bar respectively. It should be noted that the tests at 1 bar and 3 bar were not carried out with the same mixture. The measurement obtained by gas chromatography analysis showed a maximum deviation lower than $3 \%$ on the equivalence ratio of each mixture.

\subsection{The chamber and its environment}

The flame propagation is observed until the chamber walls through the $360^{\circ}$ fused silica ring. For the current study, direct flame chemiluminescence visualisation was adopted. The accurate flame front position determination will be assessed later.

The spherical geometry of the external face of the $360^{\circ}$ transparent ring was conditioned by our desire to limit optical deformations. However, even with this precaution, it is necessary to perform an optical calibration of the system. The projection of a sphere on a screen presents a difficulty. The scaling problem is that a sphere relatively close to the viewpoint presents a visual angle that is larger than the visual angle of its physical diameter. In other words, projecting the diameter of a sphere in perspective space will overestimate its actual apparent size as illustrated in Figure 3-a.
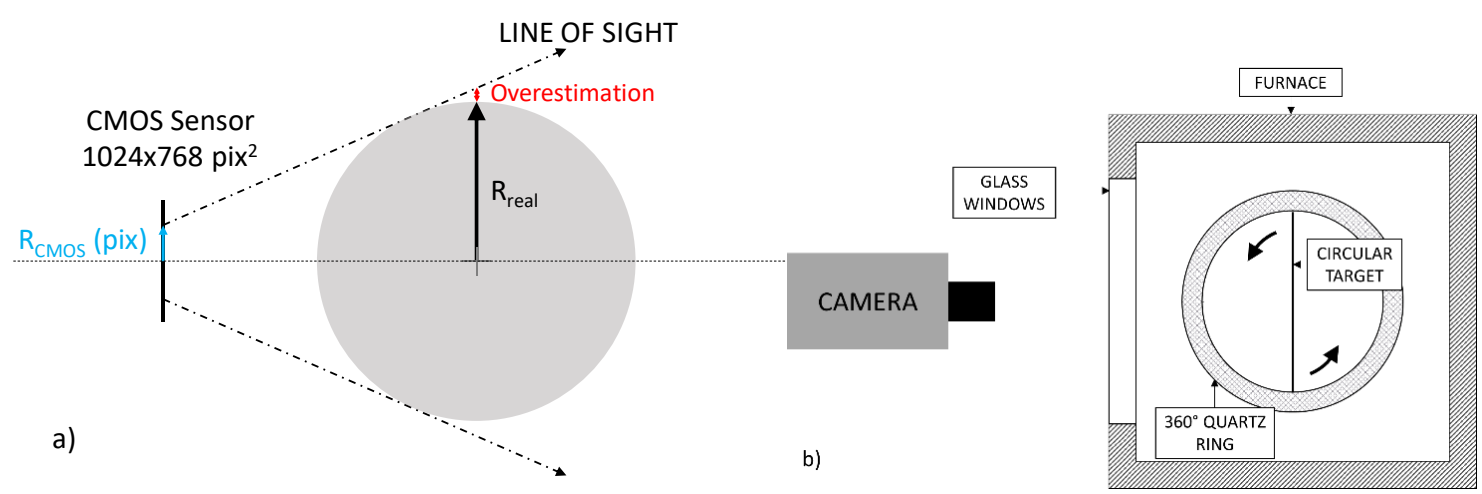

Figure 3 : a) Illustration of the projection issue -b) Illustration of the calibration procedure

The difference between the observed diameter $\left(R_{\text {real }}+\right.$ Overestimation $)$ and the real one $\left(R_{\text {real }}\right)$ increases as the diameter of the flame increases, to reach a maximal deviation of around $3 \%$ for the largest radii. In the present configuration, a simplified equation cannot be used as the fused silica ring and the glass window of the oven are between the spherical flame and the screen (see Figure 3-b). To handle this scaling problem, an in-situ calibration was performed. This procedure is detailed in supplementary material (SM1_Calibration-Procedure). It is almost equivalent to the visualization by the camera of different spheres of increasing diameters. The calibration process aims at determining the relation between the real flame radius $\left(R_{f}(\mathrm{~mm})\right)$ and the apparent flame radius observed on the CMOS chip $\left(R_{\text {CMOS }}(\right.$ pix. $\left.)\right)$ for all flame dimensions: $R_{f}(m m)=f\left(R_{C M O S}(\right.$ pix. $\left.)\right)$.

Based on experimental calibration, a quadratic polynomial fit was inferred with a good coefficient of determination $\left(R^{2}>0.99\right)$ to recover $R_{f}(\mathrm{~mm})$ based on $R_{C M O S}$ (pixel) measurement. The error made on the flame radius determination was quantified and corresponds to a subpixel offset. 


\subsubsection{Flame radius determination}

The acquisition rate was adjusted for each case to allow an accurate description of the flame front propagation (i.e. 6000 or $10000 \mathrm{fps}$ ). The camera electronic shutter was set to the minimum value allowing a sufficient flame detection during all the propagation (i.e. $<30 \mu \mathrm{s}$ for all flame conditions), ensuring that the flame front displaces for largely less than one pixel during this time. The blurring effect due to the exposure time is negligible. The resolution of the CMOS sensor was set to $1024 \times 768$ pixels ${ }^{2}$ with a magnification ratio $\sigma=0.124 \mathrm{~mm} /$ pix.

The flame detection is based on the recording of the direct flame emission. One source of flame emission is chemiluminescence, the electromagnetic radiation emitted from the de-excitation of electronically excited species that are formed via chemical reactions in the flame front. $\mathrm{CH}^{*}, \mathrm{C}_{2}^{*}, \mathrm{OH}^{*}$ and $\mathrm{CO}_{2}^{*}$ are responsible for much of the visible and ultraviolet chemiluminescence in typical hydrocarbon-air flames [39]. $\mathrm{CO}_{2}^{*}$ background is present in the $200-600 \mathrm{~nm}$ region $[40,41]$. As a result, for methane-air flames with equivalence ratio ranging from 0.8 to 1.3 , the major contribution of the integrated visible chemiluminescence comes from $\mathrm{CO}_{2}^{*}$ as detailed in [42].

From the flame chemiluminescence recording, the typical radius extraction procedure involves edge detection and fitting method following the sequence:

- 1. Image processing and edge detection. First, a cleaning of the image is performed (background subtraction). Then, a global threshold is applied consisting of searching the greyscale space for a given value that generates a phase whose boundary coincides with the largest gradients in the original image.

- 2. Flame radius evaluation based on the surface of the binarized image. When the flame radius is smaller than $2 \mathrm{~cm}$, the fitting procedure uses a circular shape whereas it considers a disk truncated of two circular segments for greater flame radii.

It was pointed out that the level of contours chosen to track the flame front might affect the measurement of the flame front propagating speed [43] as the flame thickness varies because of varying stretch effects and changing unburnt gas thermodynamic conditions. In a previous study [44], the correct procedure for the identification of the flame location was outlined when either shadowgraph or Schlieren is implemented. In our configuration, the radius detection is based on the integrated flame chemiluminescence. The current methodology matches the position of the flame radius with the maximal gradient of the flame chemiluminescence projected on a $2 \mathrm{D}$ screen. The determination of the equivalent flame radius is based on the surface detection. The annular window allows to visualize the flame front until it reaches the chamber wall in the horizontal direction $\left(R_{0}=60.65 \mathrm{~mm}\right.$ ) but limits the visualisation vertically to around $40 \mathrm{~mm}$ (a bit less than the height of the fused silica ring to avoid edge effects). This geometry allows to detect at most a fifth of the flame edge points for the largest flame radii. Two different criteria were introduced to overcome this limitation: evaluation of both an index of sphericity error and of the displacement of the flame center. For the first criterion, based on the visible flame edges, we first find the largest sphere that can be inscribed in the data points. The center of this sphere is then used to find the smallest circumscribed sphere. The difference in the radius of these spheres defines our sphericity error. A maximal difference in the two radii of one pixel was selected. This corresponds, for large flame radii (i.e. the critical conditions in terms of flame edge points), to a sphericity index greater than 0.998 . This criterion was selected to ensure the validity of the hypothesis of sphericity, which is essential for the application of the method. In addition, an estimate of the uncertainty in the flame radius was estimated as done in 
Xiouris et al. [4] using the following formula: $a_{R_{f}}=\sqrt{\frac{\sum_{i=1}^{k}\left(r_{i}-r_{f i t}\right)^{2}}{k-1}}$, where $r_{i}$ is the distance of every edge point $i$ from the fit-obtained circle center, and $r_{f i t}$ is the fit-obtained circle radius. This error evaluation is based on around 400 points, which allows a convergence of the estimate. The uncertainty in the flame radius was estimated to be lower than one pixel during the flame propagation. Then, the position of the flame center was also scrutinised during the flame propagation. As previously mentioned, the method relies on a perfect sphericity of the flame but also on a central ignition and an isotropic propagation. This is essential to ensure that the flame reaches the chamber wall at the same moment in all directions. Knowing the exact position of the center of the chamber, a maximum shift of 2 pixels (i.e. $0.24 \mathrm{~mm}$ ) is tolerated to stop the post-processing in case of flame ball convection. This will affect the last stages of the flame propagation and its interaction with the wall but not the flame radius detection as the center position is left free. Note that the flame radius evaluation is stopped well before the end of the adiabatic regime (this will be discussed later).

To identify the selected position in the flame, flame structures were computed first using CHEMKIN PREMIX [45]. The GRI Mech 3.0 [46] is the baseline mechanism used in our calculations. The chemistry of $\mathrm{CO}_{2}^{*}$ was incorporated based on the work of Kopp et al. [47]. For both mixture conditions ignited at atmospheric pressure, it was demonstrated (see supplementary material: SM2_Flame-FrontDetection) that the flame radius experimentally detected corresponds to the position of the maximum heat release during the whole propagation, with an excellent agreement (offset lower than one tenth of a pixel). As increasing initial thermodynamic conditions results in a flame thickness reduction, the tests at $P_{0}=3 \mathrm{bar} \& T_{0}=404 \mathrm{~K}$ are even more favourable. We can conclude that the flame radius extraction using the direct flame chemiluminescence is a good indicator, in our conditions and with our experimental imaging resolution, of the maximum heat release position.

The considerations discussed above allow the evaluation of a global uncertainty associated with the experimental flame radius determination. Five different contributions have been identified: 1) the optical calibration with a subpixel precision, 2) the blurring effect due to the CMOS exposure time with a subpixel precision, 3) the detection of the non-sphericity of the flame and the associated tolerated deformation, 4) the uncertainty in the flame radius lower than 1 pixel 5) the maximum flame center shift of 2 pixels which does not affect the flame radius evaluation, and 6) the flame radius extraction using the direct flame chemiluminescence with a subpixel precision. Based on these different contributions, a global precision of \pm 2 pixels was evaluated. With the flame radius ranging from $1 \mathrm{~cm}$ to $6.065 \mathrm{~cm}$, this leads to a relative precision of $2.5 \%$ in the initial stage of the flame propagation to less than $0.5 \%$ as the pressure increases $\left(\frac{P}{P_{0}}>2\right)$.

\subsubsection{Flame radius evolution}

Flame propagation visualisation of the atmospheric stoichiometric condition is illustrated in Figure 4. The left and right red arcs delimit the inner surface of the combustion chamber. Thanks to the $360^{\circ}$ fused silica ring, the flame visualisation is possible until the flame reaches the wall. The plane in which the electrodes are located is tilted to be perpendicular to the viewing axis of the camera. As illustrated on the different images of the sequence, the flame is perfectly spherical during the whole process. No instability occurs on the flame surface. Note that the development of instabilities can be detected with this optical diagnostic and will be discussed in section 3.1.3. The $360^{\circ}$ fused silica ring is $50 \mathrm{~mm}$ height. The consequence is that the flame visualisation is limited in the vertical direction to a maximal radius of $2 \mathrm{~cm}$ (upper and lower red limits reported in Fig. 4). This limit is reached before $11 \mathrm{~ms}$ under these conditions. Then, the flame detection is based on the disk truncated of two circular segments. The flame luminosity seen by the CMOS sensor increases with the flame radius. This requires adjusting the 
exposure time to achieve a good compromise between good detection of small flames radii and avoid image saturation for large flame radii.

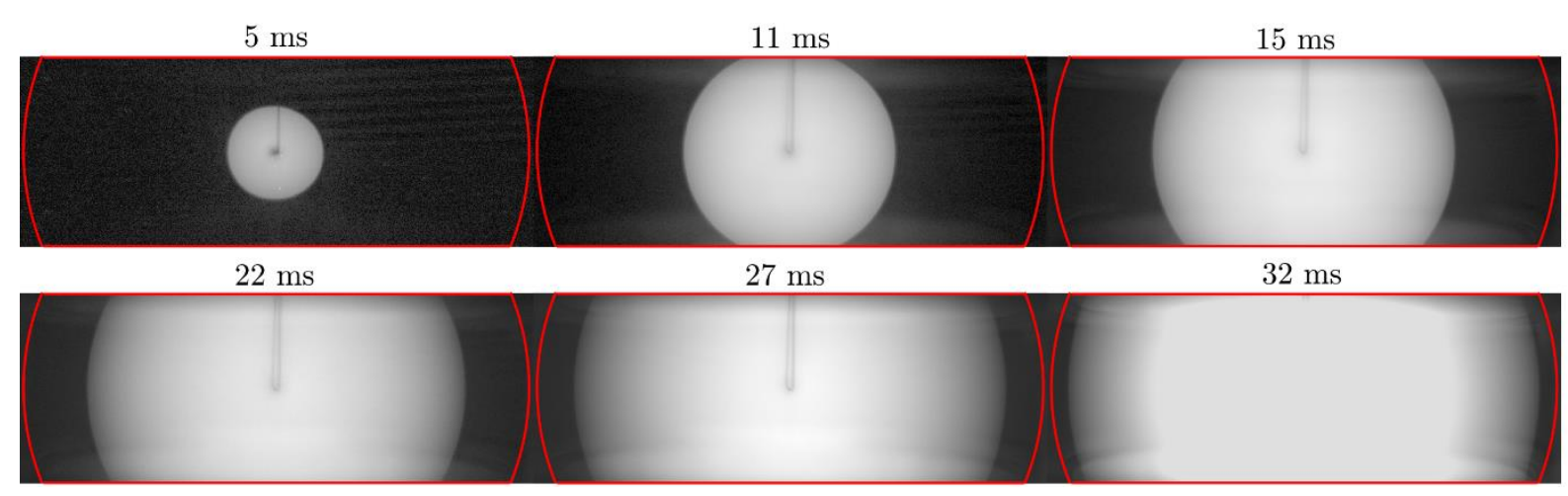

Figure 4 : Sequence of flame chemiluminescence visualisation in the combustion chamber; stoichiometric $\mathrm{CH}_{4} /$ air flame; $P_{0}=$ 1 bar $\& T_{0}=300 \mathrm{~K}$. Red contours indicate the inner diameter of the $360^{\circ}$ quartz ring and the upper and lower limits.

The high acquisition rate of the camera allows an accurate tracking of the flame front. The temporal evolution of the flame radius is plotted in Figure 5 for the two mixture conditions $(\phi=1.0 \& 1.3)$ and for the two initial $P \& T$ conditions. The fastest propagations correspond to the stoichiometric conditions (solid and dashed red lines). The radius evolutions illustrated in Figure 5 was converted to centimeters using the optical calibration. The $y$-axis limit has been set to $6.065 \mathrm{~cm}$, which corresponds to the chamber inner radius. It is important to note that the triggering of the camera and the pressure acquisition start simultaneously when the discharge occurs in the ignition coil. Note that with the elevated acquisition rates, each flame propagation is described with at least 300 time steps.

For the atmospheric stoichiometric mixture (solid red lines), nine consecutive flame propagations were acquired, keeping constant the initial pressure and temperature $\left(P_{0}=1 \mathrm{bar} \& T_{0}=300 \mathrm{~K}\right)$. The mixture composition is exactly the same as it is preliminary prepared in the high-pressure buffer tank. The nine evolutions are plotted in Figure 5. They are perfectly superimposed, illustrating the accuracy of the radius extraction procedure as well as the high-repeatability of experiments. As the camera exposure time is adjusted to avoid the saturation of the CMOS at large flame radii, the initial stages of the flame propagation are more difficult to detect, which explains the lack of data for flame radii lower than $5 \mathrm{~mm}$. This poses no particular problem since this phase of isobaric propagation is not of great interest in this current work, where we are mostly interested in the non-isobaric propagation. In addition, the initial propagation is more strongly affected by ignition and stretch effects.

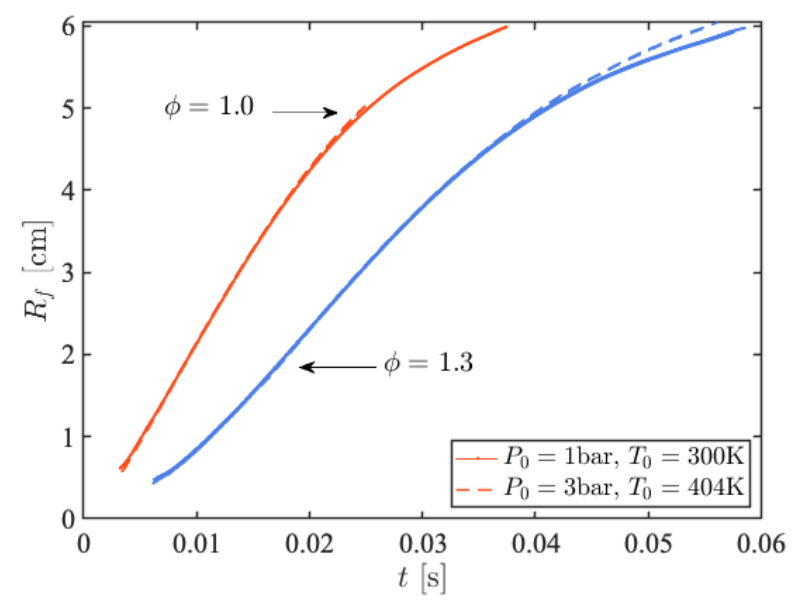

Figure 5: Time evolution of the experimental flame radius for the four $\mathrm{CH}_{4} /$ Air mixtures $(\phi=1.0$ in red $-\phi=$ 1.3 in blue; solid lines for $P_{0}=1$ bar $\& T_{0}=300 \mathrm{~K}$ - dashed lines for $P_{0}=3$ bar \& $T_{0}=404 \mathrm{~K}$ ). 
For the atmospheric fuel-rich condition, four experiments were performed and their results are plotted in solid blue lines in Figure 5. Again, the experimental repeatability is excellent. The initial flame propagation is delayed because of stretch effects and a lower flame reactivity. For $P_{0}=3 \mathrm{bar} \& T_{0}=$ $404 K$, the flame propagations are similar to their associated atmospheric conditions exhibiting the pressure and temperature counterbalancing effects. Three experiments were performed for each mixture. For $\phi=1.3$, smooth stable spherical flame was always observed. However, for the stoichiometric condition the flame radius temporal evolution was plotted only for $R_{f}<5.05 \mathrm{~cm}$ due to the occurrence of instability on the flame surface (see Section 3.1.3).

\subsubsection{Pressure trace}

Pressure traces were acquired at a frequency of $20 \mathrm{kHz}$ using two identical pressure transducers located respectively on the upper and lower part of the chamber. The two transducers are connected to charge amplifiers (AVL FI Piezo amplifier module 2P2G) linked to an acquisition system (eight channels, 16 bits and $200 \mathrm{kS} / \mathrm{s}$ per channel). The linearity of the sensor is $\pm 0.3 \%$ of the full-scale range. For an initial pressure of $1 \mathrm{bar}$, the range of the pressure sensor was set to be a maximal pressure of 10 bars, leading to the accuracy of \pm 0.03 bar.

The two sensors are subject to the same pressure at the same moment. The use of two opposed transducers was envisaged here to ensure no shifting of one of them. When the flame reaches the wall, the experimental pressure trace departs from the simulated one due to heat losses [22]. Comparison of the two experimental traces during this phase may be a good indicator of the central ignition and isotropic flame propagation (i.e. no buoyancy effect).

Similarly to the flame radius, nine and four tests are reported for the atmospheric stoichiometric and fuel-rich conditions respectively, and three for the high-pressure conditions. Normalized pressure traces are reported in Figure 6. They were deliberately truncated before flame instability occurs (red dashed lines) or the flame reaches the wall (the other three lines). Pressure traces are perfectly smooth and no pressure oscillations are detected as this can be observed in a rectangular chamber[48].

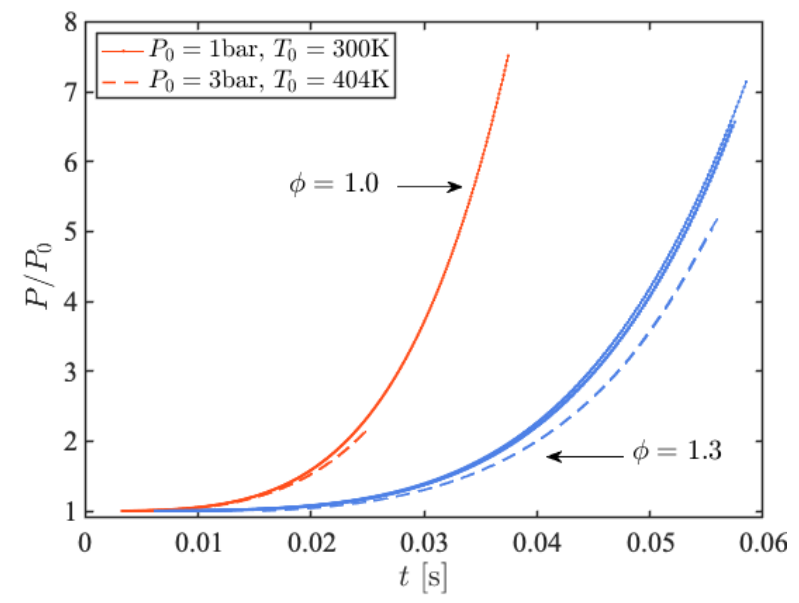

Figure 6: Normalized pressure traces for the four $\mathrm{CH}_{4} /$ Air mixtures $(\phi=1.0$ in red and $\phi=$ 1.3 in blue; solid lines for $P_{0}=1$ bar \& $T_{0}=300 \mathrm{~K}$-dashed lines for $P_{0}=3$ bar \& $\left.T_{0}=404 \mathrm{~K}\right)$.

To complete the information provided by Figure 5 and Figure 6 , variations of $R_{f}$ with $P$ $\left(R_{f}=f\left(P / P_{0}\right)\right)$ are plotted in Figure 7 as done in $[4,21]$.

Combining Eq. (3) and the simple linear $x(P)$ relation initially derived by Lewis and Von Elbe [11] yields the following relation: 
$\frac{R_{f}}{R_{0}}=\left[1-\left(1-\frac{P-P_{0}}{P_{e}-P_{0}}\right)\left(\frac{P_{0}}{P}\right)^{1 / \gamma_{u}}\right]^{1 / 3}$

As discussed by Chen et al. [21], the $R_{f}=f(P)$ profile depends on the equilibrium pressure $P_{e}$ and the heat capacities ratio of unburnt gas $\gamma_{u}$. Under our conditions, it is independent, for each initial pressure condition, of the magnitude of $d P / d t$ (and so of the flame speed $S_{u}$ ) [8].

For $\mathrm{CH}_{4}$ /air mixtures, both the equilibrium pressure $P_{e}$ and the heat capacities ratio of unburnt gas $\left(\gamma_{u}\right)$ for the stoichiometric condition are very close to those at $\phi=1.3$ (the relative difference is within $0.2 \%)$. Therefore, according to Eq. (6) the $R_{f}-P$ curves for these two mixtures are almost superimposed as illustrated in Figure 7.

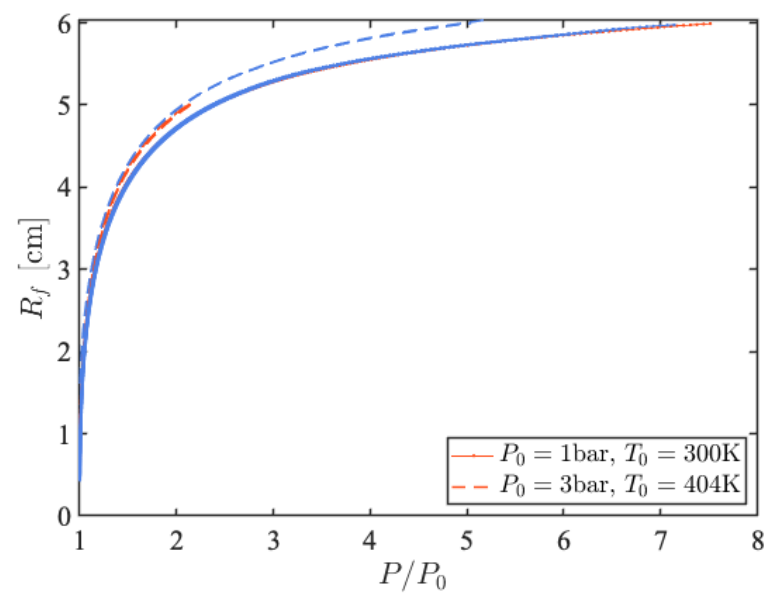

Figure 7 : Variation of $R_{f}$ with $P$ for the four $\mathrm{CH}_{4} /$ Air mixtures $\left(\phi=1.0\right.$ in red and $\phi=1.3$ in blue; solid lines for $P_{0}=$ 1 bar $\& T_{0}=300 \mathrm{~K}-$ dashed lines for $P_{0}=3$ bar $\left.\& T_{0}=404 \mathrm{~K}\right)$.

Note that all the flame radius and pressure raw data are included in supplementary material (SM3_Data).

\section{Results and discussion}

\subsection{Limits of the method}

\subsubsection{Radiation and wall boundary effects}

The flame propagation in the spherical chamber is influenced by several parameters. Numerical results could afford valuable guidelines to evaluate both radiation and wall boundary influence on the flame speed.

Numerical simulations were performed with either adiabatic or isothermal walls. For isothermal walls, the wall temperature is the same as the initial temperature of unburned gas, and three different gas radiation models were tested: an adiabatic model with no radiative loss (ADI), an optically thin model considering emission but no absorption (OTM) and a statistical narrow band model with both radiation emission and absorption (SNB). These three models are described in more details in Chen et al. [49].

The flame speed evolutions are reported in Figure 8. For these two mixture conditions, isothermal results obtained without radiation (ADI) and with radiation with reabsorption (SNB) are very close, indicating that flame speed is slightly affected by radiation. Moreover, wall boundary effects are very weak for $P<6.5$ bar for both equivalence ratios. 


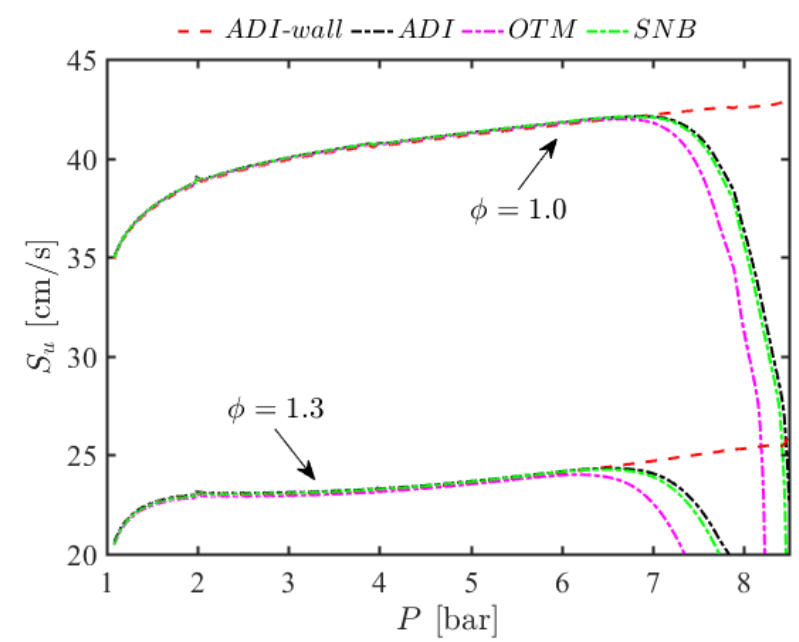

Figure 8: DNS results of flame speed evolution with pressure for adiabatic walls (dashed red line) and isothermal walls (dotdash lines) with different radiation models with GRI Mech 3.0: no radiative loss (ADI), optically thin model (OTM), statistical narrow band model (SNB); $\phi=1.0$ (in upper part) and $\phi=1.3$ (in lower part) ; $P_{0}=1$ bar \& $T_{0}=300 \mathrm{~K}$.

This information allows defining the experimental data range to consider. As illustrated in Figure 9 and explained in Omari et al. [22], the time derivative of the pressure trace is a good indicator of the heat losses to the wall. This criterion is also used by Burrell et al. [13].Comparing the flame propagation with and without heat losses to the wall points out a limit to consider, which corroborates the value of 6.5 bars previously found. Experimental traces of time pressure derivatives are also plotted in Figure 9 for the two mixture conditions (solid lines; red for $\phi=1.0$ / blue for $\phi=1.3$ ). The pressure corresponding to the maximum of $\frac{d P}{d t}$ is identified and based on DNS results, a criterion of $90 \%$ of $\max \left(\frac{d P}{d t}\right)$ is introduced to define the maximal experimental pressure to consider. This criterion ensures that no wall effect can occur during the propagation investigated. This leads to consider, for the rest of the paper, an upper pressure value of 6 bar for $\phi=1.0 \& 1.3$ and $P_{0}=1$ bar \& $T_{0}=$ $300 \mathrm{~K}$.

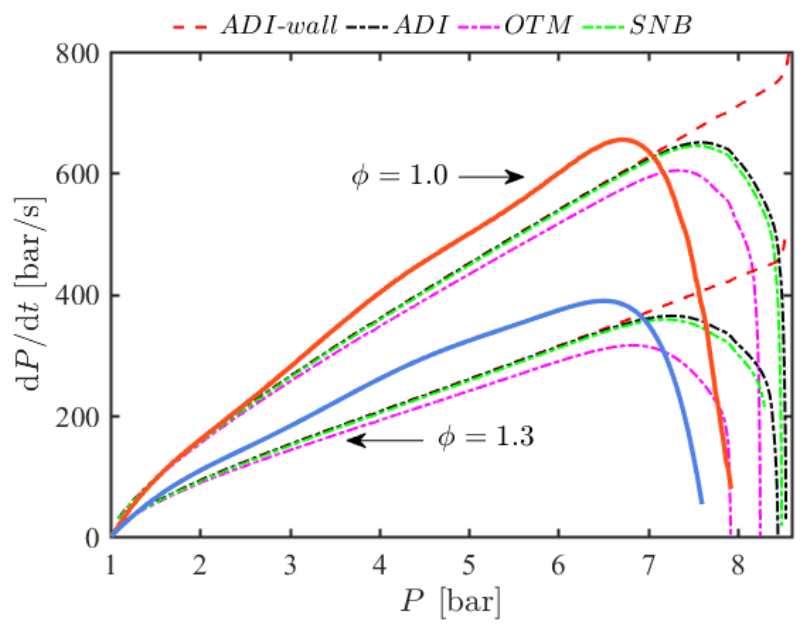

Figure 9 : Time derivative of pressure traces for adiabatic walls (dashed red lines) and isothermal walls (dot-dash lines) with different radiation models with GRI Mech 3.0. Solid lines for experimental results; $\phi=1.0$ (in upper part) and $\phi=1.3$ (in lower part) $; P_{0}=1 \mathrm{bar} \& T_{0}=300 \mathrm{~K}$.

Eq. (2) considers an isentropic compression of the unburnt gas. The validity of this assumption was tested for the stoichiometric condition comparing the simulated temperature evolution of unburnt gas (DNS) to the one evaluated using an isentropic compression hypothesis. The heat capacities ratio of 
unburnt gas $\left(\gamma_{u}\right)$ was evaluated based on thermodynamic data. It resulted from this comparison that the compression can be considered as isentropic in the pressure range previously defined $(P \epsilon[1-6]$ bar $)$, confirming the conclusions drawn from the pressure derivative evolution. This is consistent since radiation and heat losses to the wall are the main potential causes of non-isentropic compression. The highest pressure (i.e. 6 bars) corresponds to an unburnt gas maximal temperature slightly lower than $500 \mathrm{~K}$. This limits the achievable experimental conditions with the selected initial atmospheric conditions.

Using a criterion of $90 \%$ of $\max (d P / d t)$, a maximum pressure of 15 bar should be considered for both mixture conditions initially at $\mathrm{P}_{0}=3$ bar $\& \mathrm{~T}_{0}=404 \mathrm{~K}$. This maximum pressure, corresponding to less than $75 \%$ of the theoretical end gas pressure reported in Table 1, allows reaching a compressed unburnt gas temperature higher than $600 \mathrm{~K}$. As illustrated in Figure 1, these relatively elevated thermodynamic conditions are beyond the conditions achievable by other methods.

\subsubsection{Stretch effects}

It is important to remind that the flame speed throughout its propagation is decreasingly affected by stretch effects. In the constant volume method, the stretch effect on the laminar flame speed might be too large to be neglected for mixtures with Lewis numbers greatly deviating from unity and thus the stretch correction for obtaining accurate unstretched laminar flame speed is indispensable [25]. Chen et al. [21] have numerically investigated stretch effects for the constant volume method for hydrogen/air, methane/air and propane/air mixtures. Experiments and DNS can be compared directly since they are both affected by stretch effect, but a problem may arise if experimental results are compared with unstretched data obtained with steady 1D simulations (using the PREMIX code for example). In order to allow, in the future, a direct comparison with steady 1D simulations, we propose to consider only conditions of low stretch in the current study.

The flame information corresponding to the ambient condition are listed in Table 2. Unstretched flame speed $\left(S_{u}^{0}\right)$ and burnt gas Markstein length $\left(L_{u}\right)$ were taken from Varea et al. [50] (the value of $L_{u}$ for the fuel-rich condition was extrapolated).

Table 2 : Flame information for the selected mixture conditions

\begin{tabular}{llllll}
\hline Fuel & $T_{0}(\mathrm{~K})$ & $P_{0}(\mathrm{bar})$ & $\phi(-)$ & $S_{u}^{0}(\mathrm{~m} / \mathrm{s})$ & $L_{u}(\mathrm{~mm})$ \\
\hline $\mathrm{CH}_{4}$ & 300 & 1 & 1 & 0.36 & -0.13 \\
$\mathrm{CH}_{4}$ & 300 & 1 & 1.3 & 0.22 & 0.3 \\
\hline
\end{tabular}

Stretch evolutions with pressure are reported in Figure 10. The stretch acting on the flame front is evaluated using: $K=\frac{2}{R_{f}} \frac{d R_{f}}{d t}$, as the flame sphericity has been checked by flame visualisation. A sharp decrease in stretch is observed for pressure between 1 and 2 bars. For $P>2$ bars, the stretch level is lower than $K<60 s^{-1}$. With the laminar conditions indicated in Table 2, and assuming a linear relation between the flame speed and the stretch $\left(S_{u}=S_{u}^{0}-L_{u} K\right)$, this leads to relative error in flame speed lower than $2 \%$, regardless of the mixture. 


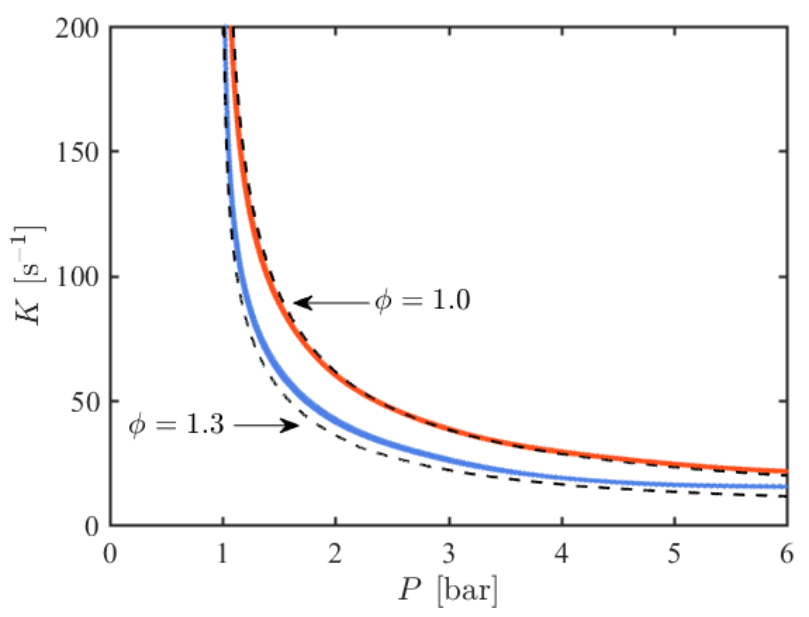

Figure 10 : Stretch evolution as a function of pressure for stoichiometric (solid red lines) and fuel rich ( $\phi=1.3$ - solid blue lines) $\mathrm{CH}_{4} /$ Air mixtures. Dashed lines correspond to DNS results with GRI Mech 3.0; $P_{0}=1 \mathrm{bar} \& T_{0}=300 \mathrm{~K}$.

The current results perfectly match the ones obtained by Chen et al. [21] and Xiouris et al. [4] where it is numerically demonstrated that stretch effects may be generally neglected for $P / P_{0}>2$ and $P / P_{0}>2.5$, respectively. In the following, the lower pressure value considered will be limited at $P / P_{0}=2$.

It is important to recall that based on the optical calibration, limiting the pressures considered to $P / P_{0}>2$ allows maintaining a precision less than $0.5 \%$ on the radius determination during the entire period of interest.

\subsubsection{Instability detection}

Some groups $[4,51]$ have developed two complementary set-ups to ensure that no instabilities develop on the flame surface during the propagation. With our configuration, OPTIPRIME, the development of wrinkles on the flame surface can be visually detected. The flame propagation of the two high-pressure conditions are illustrated in Figure 11. The timing corresponding to the different images is also indicated. The four pairs of images $(a-\alpha, b-\beta, c-\gamma, d-\delta)$ correspond to equivalent flame radii. It can be seen from this figure that the flame front for the fuel-rich mixture (left-hand side) is perfectly smooth and spherical until the flame reaches the wall. On the contrary, the presence of a first wrinkle in Figure 11-a, 19 ms after the ignition, can clearly be detected. After $25 \mathrm{~ms}$ of propagation, the amplitude of the initial wrinkle amplifies and $2 \mathrm{~ms}$ later, the whole flame surface is covered by cells.

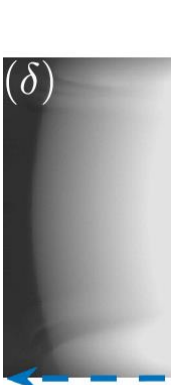

$50 \mathrm{~ms}$ $\phi=1.3$

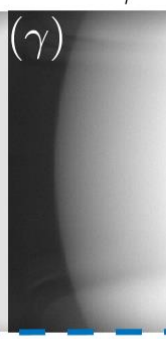

$45 \mathrm{~ms}$

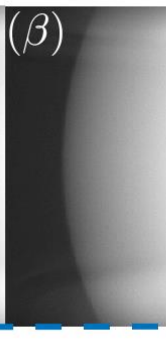

$41 \mathrm{~ms}$

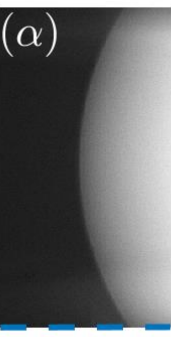

$32 \mathrm{~ms}$

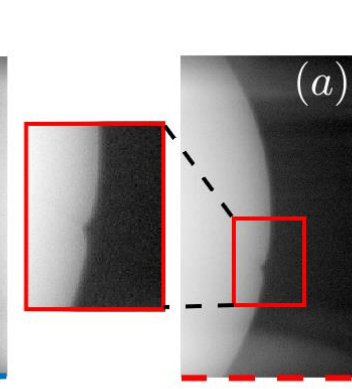

$19 \mathrm{~ms}$ $\phi=1.0$

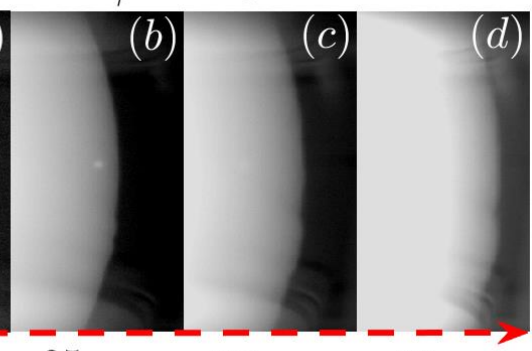

$25 \mathrm{~ms}$

$27 \mathrm{~ms}$

$30 \mathrm{~ms}$

Figure 11 : Sequences of flame chemiluminescence visualisation in the combustion chamber; $P_{0}=3$ bar \& $T_{0}=404 K$. Left (blue frame): fuel-rich condition- Right (red frame): stoichiometric $\mathrm{CH}_{4} /$ air flame 
As illustrated in Figure 11, OPTIPRIME allows detecting the occurrence and propagation of cells on the flame surface, simultaneously with pressure and radius acquisition. This unique performance represents a tremendous advantage for the constant volume method.

\subsection{Performances of the new proposed method}

The new proposed methodology relies on an accurate simultaneous measurement of pressure and flame radius evolutions. Its ultimate goal is to evaluate, using equation 2 , flame speed evolution during the compression and to use it as a target for kinetic schemes validation. This is not done in the current paper which only focuses on the metrics measurable with the new experimental system. The precision associated with the flame radius has been carefully described previously and it was concluded that a maximal error of \pm 2 pixels can may be achieved with the current system. In the following, we propose to investigate how this level of accuracy compares to those obtained applying traditional methods. A complete comparison would need to perform simulations using experimental pressure traces and a thermodynamics-based model including radiation effects [4]. Unfortunately we do not have this tool. Thereby we have performed direct numerical simulations to evaluate the impact of radiation on the radius determination in the case of the most critical condition (3bar, 404K, fuel rich condition). This allows evaluating the relative error that could be linked to radiation effects and how this uncertainty compares to the one obtained with new proposed methodology.

The evaluation of the burnt mass fraction $(x)$ at any time during flame propagation is the weak point of the algebraic models. As it cannot be measured directly, it is related to the pressure rise inside the vessel (see equations 4 and 5). This is briefly discussed in the introduction and detailed in Faghih and Chen [9]. The performances of the new experimental method are compared, in terms of accuracy to three different models: the original model from Lewis and von Elbe [11], the modified model proposed by Luijten et al. [23] and the adjusted model proposed by Faghih and Chen [9], which is one of the most recent. These three models are summarized in Table 3.

Table 3 : Equations used to determine the burnt mass fraction, $x$

\begin{tabular}{|c|c|}
\hline Group & Correlation \\
\hline Lewis and von Elbe [11] & $x=\frac{\left(P-P_{0}\right)}{\left(P_{e}-P_{0}\right)}$ \\
\hline Luijten et al. [23] & $f(P)=\frac{\gamma_{b}-1}{\gamma_{u}-1}+\frac{\gamma_{u}-\gamma_{b}}{\gamma_{u}-1}\left(\frac{P}{P_{0}}\right)^{\left(\gamma_{u}-1\right) / \gamma_{u}}$ \\
\hline Faghih and Chen [9] & $x=\frac{P-P_{0} \cdot f(P)}{P_{e}-P_{0} \cdot f(P)}, \quad f(P)=\frac{\gamma_{b}-1}{\gamma_{u}-1}+\frac{\gamma_{u}-\gamma_{b}}{\gamma_{u}-1}\left(\frac{P}{P_{0}}\right)^{\left(\gamma_{u}-1\right) / \gamma_{u}}, \gamma_{b, \text { shift }}=\frac{\gamma_{b}+8}{8}$ \\
\hline
\end{tabular}

The flame radius experimental trace is set as the reference and its associated level of precision is reported. Figure 12 illustrates the performances of the three models showing the percentage difference reflected in $R_{f}$ when comparing the simulated results to the experimental accuracy. The latter is represented symmetrically in dashed black lines with an initial value of $0.5 \%$ for $\frac{P}{P_{0}}=2$ decreasing to $0.4 \%$ for $\frac{P}{P_{0}}=6$. 
(a) $: \phi=1.0$

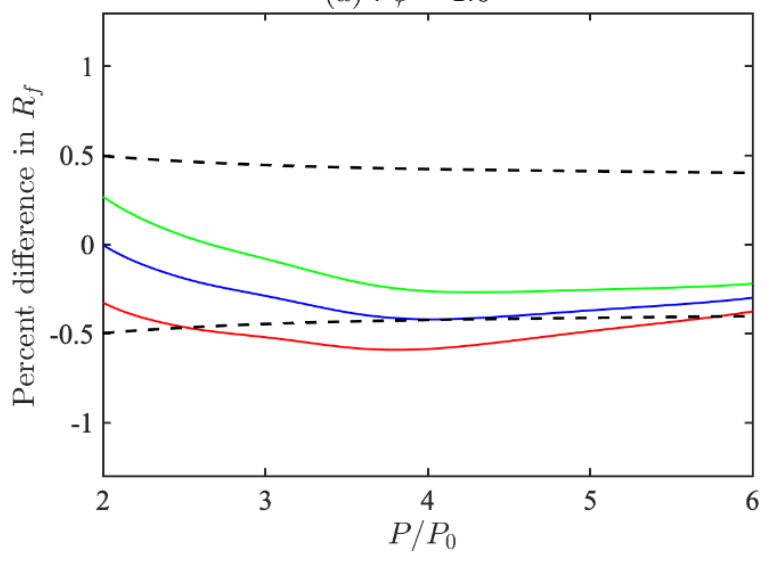

$(b): \phi=1.3$

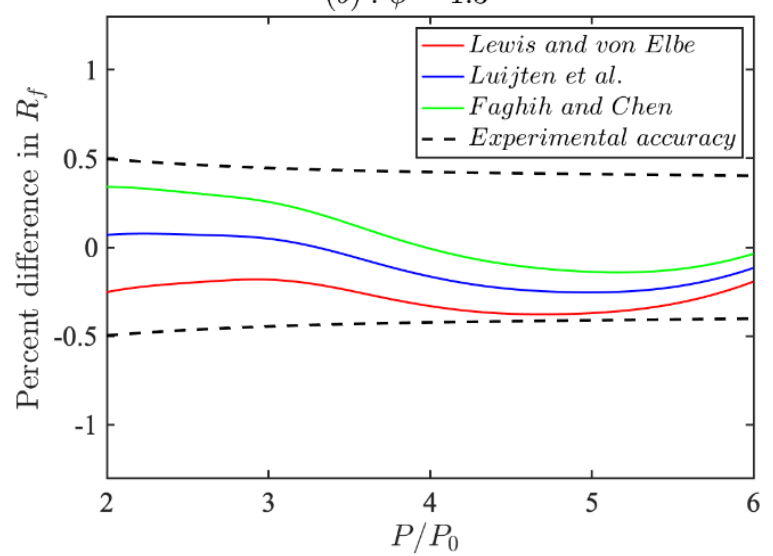

Figure 12 : Percent difference in $R_{f}$ calculated with the three models of Table 3 and compared to the experimental accuracy (black dashed lines) for $\mathrm{CH}_{4} /$ air flame with $P_{0}=1$ bar $\& T_{0}=300 \mathrm{~K}$. Left (a): stoichiometric - Right (b): fuel-rich.

The use of the different models results in a global accuracy comparable to the experimental one under atmospheric conditions. These three models afford comparable values with slight differences more pronounced in the initial stage of the flame propagation. For the tested conditions, the new proposed methodology, requiring a precise flame radius measurement, afford a level of accuracy comparable to standard algebraic models.

The models are now tested under fuel-rich condition at $\mathrm{P}_{0}=3$ bar $\& \mathrm{~T}_{0}=404 \mathrm{~K}$. Results are reported in Figure 13. The stoichiometric condition was not always stable and thereby is not considered there. The experimental accuracy remains unchanged and is still represented by the symmetrical black dashed lines. However, the accuracy of the three models is observed to be much lower (between 2 and $3 \%)$.

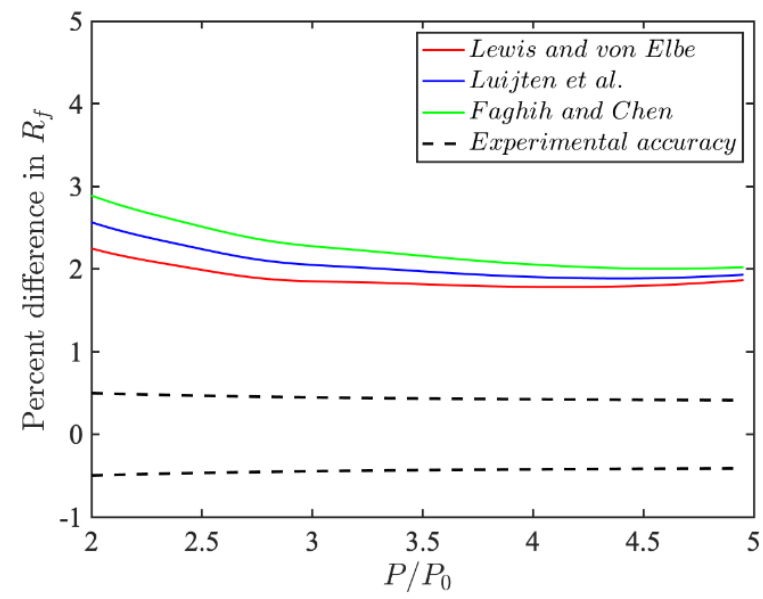

Figure 13 : Percent difference in $R_{f}$ calculated with the three models of Table 3 and compared to the experimental accuracy (black dashed lines); fuel rich condition ; $P_{0}=3$ bar $\& T_{0}=404 \mathrm{~K}$.

Xiouris et al. [4] have evaluated the effect of radiation on the flame propagation using experimental pressure traces and thermodynamic models for different mixtures at thermodynamic states relevant to engines conditions. They have shown that neglecting radiation in constant volume experiments could introduce, for their conditions, errors in radius of $1.5 \%$. Although their experimental conditions are different from ours, this affords a relevant information knowing that the uncertainty of our methodology is independent of the conditions and always lower than $\pm 0.5 \%$. 
Additional DNS calculations were performed to evaluate the impact of radiation for our most critical condition ( $P_{0}=3 \mathrm{bar} \& T_{0}=404 \mathrm{~K}$, fuel rich condition). This allows evaluating the minimal relative error that would be obtained using a thermodynamic model. The SNB model is selected as the reference since it is the most realistic (including both radiation emission and absorption). The relative difference in flame radius is evaluated considering successively no emission with isotherm (ADI-wall) or adiabatic (ADI) walls and then emission but no absorption (OTM). The relative differences are reported in Figure 14. The percent difference in flame radius relates to heat losses is of the order of $\pm 0.5 \%$, which is comparable, for this condition, to the experimental accuracy.

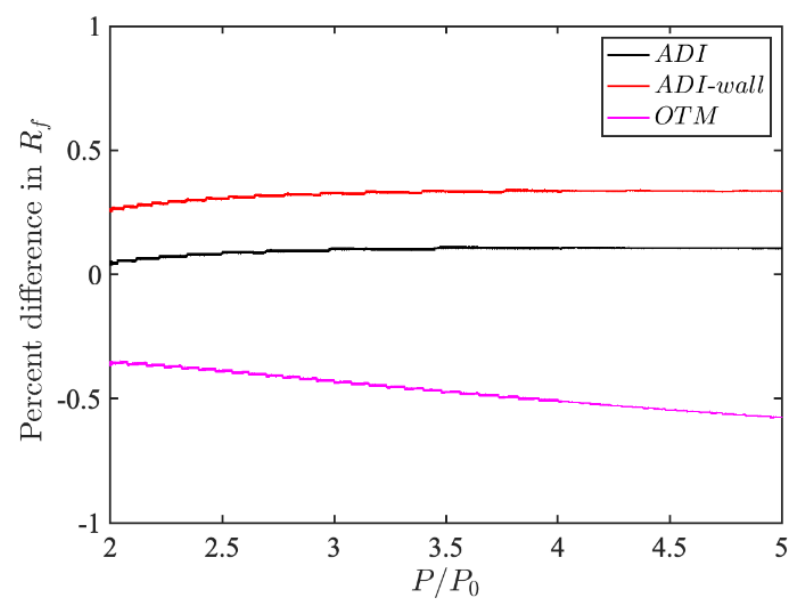

Figure 14: Percent difference in $R_{f}$ calculated with different radiation models (ADI, ADI-wall, OTM) and compared to the most realistic model (SNB); fuel rich condition ; $P_{0}=3$ bar \& $T_{0}=404 \mathrm{~K}$.

One can legitimately wonder if the gain in precision, of a few percents, corresponds to a real progress. The flame speed may be evaluated using Eq. (2). This equation was used to estimate the sensitivity of the flame speed to the three variables: flame radius $\left(R_{f}\right)$, pressure $(P)$ and unburnt gas heat capacities ratio $\left(\gamma_{u}\right)$. Note that we do not propagate here the uncertainty in the derivatives $d R_{f} / d t$ and $d P / d t$. This was done carefully in Xiouris et al. [4]. A local polynomial fitting procedure was applied to reduce the associated error. For a global uncertainty evaluation, which is out of the scope of the current work, their careful investigation may be considered as a guideline.

A $2 \%$ perturbation was imposed separately on each of the three variables and the resulting flame speed variation, represented by $S_{u} / S_{u, \text { original }}$, is reported in Figure 15 . The sensitivity analysis was performed for the stoichiometric atmospheric condition but all cases lead to similar conclusions.

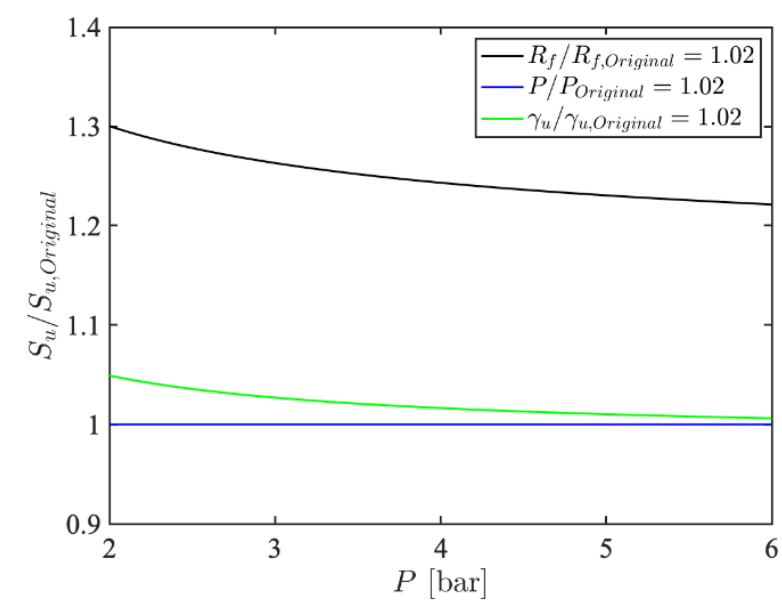


Figure 15 : Sensitivity analysis of the flame speed to the flame radius $\left(R_{f}\right)$, the pressure $(P)$ and the unburnt gas heat capacities ratio $\left(\gamma_{u}\right)$; stoichiometric $\mathrm{CH}_{4} /$ air flame; $P_{0}=1$ bar $\& T_{0}=300 \mathrm{~K}$.

Figure 15 clearly demonstrates that the flame speed is extremely sensitive to the flame radius determination. Indeed, a small initial disturbance is amplified by a factor of 10 . This ties in with the conclusions of Jayachandran et al [4] who already pointed out that the extracted flame speed is very sensitive to $R_{f}(P)$. Figure 15 also shows that the flame speed is sensitive to $\gamma_{u}$ to a much lesser extent and virtually insensitive to pressure.

To generalize these conclusions, the amplitude of the initial perturbation was varied until $5 \%$ as illustrated in Figure 16. For this comparison, the value of $S_{u} / S_{u, \text { Original }}$ corresponding to $\frac{P}{P_{0}}=4$ was arbitrary selected.

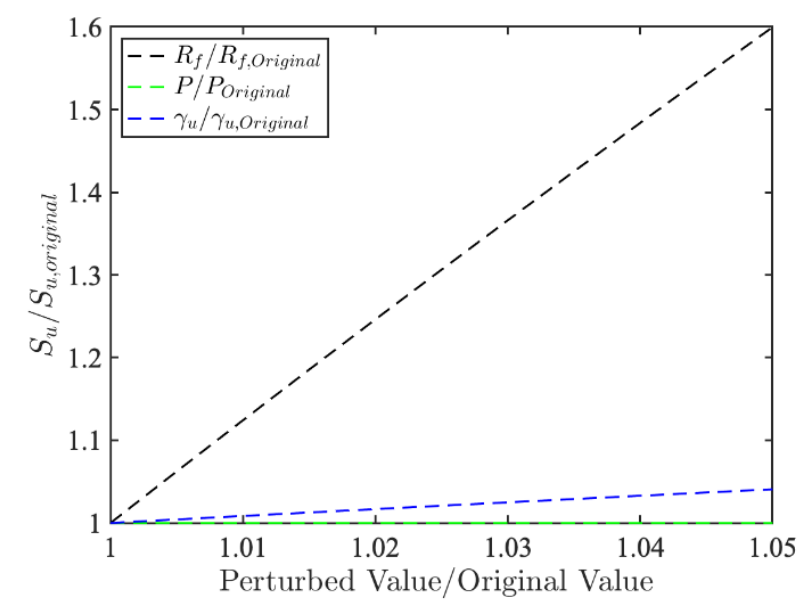

Figure 16 : Flame speed sensitivity to the amplitude of the initial perturbation of the flame radius $\left(R_{f}\right)$, the pressure $(P)$ and the unburnt gas heat capacities ratio $\left(\gamma_{u}\right)$; stoichiometric $\mathrm{CH}_{4}$ /air flame; $P_{0}=1$ bar \& $T_{0}=300 \mathrm{~K}$.

The obtained linear evolutions confirm the important sensitivity of the flame speed to the flame radius. Figure 16 indicates that 2-3\% uncertainty on the measurement of the flame radius yields $20-30 \%$ uncertainty on the evaluation of the laminar burning velocity.

As a conclusion, using the pressure trace as an input limits the propagation of uncertainties but the precision of the $R_{f}(P)$ model is clearly an issue. We have shown that available models behave very well for methane/air mixtures at atmospheric pressure, however, important inaccuracies appear for higher thermodynamic conditions as shown in Figure 13. For the current set-up, OPTIPRIME, the $R_{f}(P)$ model is not needed since the flame radius evolution can be accurately measured simultaneously with the pressure history. Consequently, the accuracy associated with the current set-up will limit the uncertainty on the flame velocity to less than $5 \%$ over the entire flame spread, whatever the conditions as long as the flame is stable. 


\section{General conclusions}

The current study describes a new experimental rig, OPTIPRIME, which consists of a spherical chamber equipped with a $360^{\circ}$ transparent ring. This system allows the simultaneous recording of the pressure inside the chamber and, fully innovative, of the flame radius until the wall. This unique system makes possible to track the flame front evolution during the whole combustion process. The direct flame visualisation is useful to ensure that the flame surface remains perfectly spherical, without being affected by instabilities or gravity effects. The flame sphericity being checked, the global stretch acting on the flame dynamics can be evaluated. A specific attention was paid to the accuracy of the flame radius measurement. A global precision of \pm 2 pixels on the flame radius has been determined. This leads to a relative precision less than $0.5 \%$ at $\frac{P}{P_{0}}>2$ and less when $\mathrm{P}$ increases.

Evaluation of the laminar burning velocity in constant volume chamber requires the knowledge of the flame radius $\left(R_{f}\right)$. Lacking this information, the burnt mass fraction $(x)$ at any time during flame propagation is mandatory. To investigate the performance of our current experimental method, three different models have been selected and tested. These three models perform well for methane/air mixtures at atmospheric pressure but are significantly less reliable for higher thermodynamic conditions. Although the accuracy of the three models reaches few percents, this results in an inaccuracy on the flame speed ten times higher. For the current set-up, the flame radius evolution can be accurately recorded simultaneously with the pressure and these models are not needed. Consequently, the accuracy associated with the new chamber limits the uncertainty on the flame velocity to less than $5 \%$ over the entire flame spread, irrespective of the conditions.

In parallel with experiments, direct numerical simulations were carried out to identify the limits of the proposed new method. Experimental data may be used for normalized pressures $\left(\frac{P}{P_{0}}\right)$ ranging from 2 to a maximal pressure corresponding to $90 \%$ of $\max (\mathrm{dP} / \mathrm{dt})$. This restrictive range allow to be unaffected by the stretch during the initial flame propagation and to avoid heat losses to the wall.

Finally, it seems useful to clarify that this approach has been developed to provide new information for heavy fuels combustion under unusual thermodynamic conditions. The investigated experimental conditions, relatively simple, were selected to allow an essential validation step. OPTIPRIME will allow major advances in the measurement of laminar flame velocity under extreme thermodynamic conditions.

\section{Acknowledgements}

Financial support from the Labex Caprysses (convention ANR-11-LABX-0006-01) and National Natural Science Foundation of China (No. 91741126) are gratefully acknowledged. We would also like to thank Florian Gourmel, Camille Marin, Maxence Laillau and Juliette Muller for their contributions. 


\section{References}

[1] T. Poinsot, Prediction and control of combustion instabilities in real engines, Proc. Combust. Inst 36 (2017) 1-28.

[2] E. Ranzi, A. Frassoldati, R. Grana, A. Cuoci, T. Faravelli, A.P. Kelley, C.K. Law, Hierarchical and comparative kinetic modeling of laminar flame speeds of hydrocarbon and oxygenated fuels, Prog. Energy Combust. Sci. 38 (4) (2012) 468-501.

[3] F.N. Egolfopoulos, N. Hansen, Y. Ju, K. Kohse-Höinghaus, C.K. Law, F. Qi, Advances and challenges in laminar flame experiments and implications for combustion chemistry, Prog. Energy Combust. Sci. 43 (2014) 36-67.

[4] C. Xiouris, T. Ye, J. Jayachandran, F.N. Egolfopoulos, Laminar flame speeds under enginerelevant conditions: Uncertainty quantification and minimization in spherically expanding flame experiments, Combust. Flame 163 (2016) 270-283.

[5] K. Kumar, C.-J. Sung, Laminar flame speeds and extinction limits of preheated $n$-decane $/ \mathrm{O}_{2} / \mathrm{N}_{2}$ and $\mathrm{n}$-dodecane $/ \mathrm{O}_{2} / \mathrm{N}_{2}$ mixtures, Combust. Flame 151 (1-2) (2007) 209-224.

[6] A.M. Ferris, A.J. Susa, D.F. Davidson, R.K. Hanson, High-temperature laminar flame speed measurements in a shock tube, Combust. Flame 205 (2019) 241-252.

[7] S.D. Tse, D.L. Zhu, C.K. Law, Morphology and burning rates of expanding spherical flames in $\mathrm{H}_{2} / \mathrm{O}_{2}$ /inert mixtures up to 60 atmospheres, Proc. Combust. Inst 28 (2000) 1793-1799.

[8] J. Jayachandran, F.N. Egolfopoulos, Effect of unsteady pressure rise on flame propagation and near-cold-wall ignition, Proc. Combust. Inst 37 (2) (2019) 1639-1646.

[9] M. Faghih, Z. Chen, The constant-volume propagating spherical flame method for laminar flame speed measurement, Sci. Bull. 61 (16) (2016) 1296-1310.

[10] M. Metghalchi, J.C. Keck, Laminar burning velocity of propane-air mixtures at high temperature and pressure, Combust. Flame 38 (1980) 143-154.

[11] B. Lewis, G. von Elbe, Determination of the speed of flames and the temperature distribution in a spherical bomb from time pressure explosion records, J. Chem. Phys. 2 (1934) 283-290.

[12] N. Hinton, R. Stone, R. Cracknell, Laminar burning velocity measurements in constant volume vessels - Reconciliation of flame front imaging and pressure rise methods, Fuel 211 (2018) 446-457.

[13] R.R. Burrell, J.L. Pagliaro, G.T. Linteris, Effects of stretch and thermal radiation on difluoromethane/air burning velocity measurements in constant volume spherically expanding flames, Proc. Combust. Inst 37 (3) (2019) 4231-4238.

[14] B. Lewis, G. von Elbe, Combustion, Flames and Explosions of Gases, Academic Press, New York 2nd edition (1961).

[15] D. Bradley, A. Mitcheson, Mathematical solutions for explosions in spherical vessels, Combust. Flame 26 (1976) 201-217.

[16] E.F. Fiock, C.F. Marvin, F.R. Caldwell, C.H. Roeder, Flame speeds and energy considerations for explosion in a spherical bomb, National advisory committee for aeronautics Report No. 682 (1940).

[17] A. Lefebvre, H. Larabi, V. Moureau, G. Lartigue, E. Varea, V. Modica, B. Renou, Formalism for spatially averaged consumption speed considering spherically expanding flame configuration, Combust. Flame 173 (2016) 235-244.

[18] A. Moghaddas, K. Eisazadeh-Far, H. Metghalchi, Laminar burning speed measurement of premixed $\mathrm{n}$-decane/air mixtures using spherically expanding flames at high temperatures and pressures, Combust. Flame 159 (4) (2012) 1437-1443.

[19] K. Saeed, C.R. Stone, The modelling of premixed laminar combustion in a closed vessel, Combust. Theor. Model. 8 (4) (2004) 721-743.

[20] C.C.M. Luijten, E. Doosje, J.A. van Oijen, L.P.H. de Goey, Impact of dissociation and end pressure on determination of laminar burning velocities in constant volume combustion, Int. J. Therm. Sci. 48 (6) (2009) 1206-1212. 
[21] Z. Chen, M.P. Burke, Y. Ju, Effects of compression and stretch on the determination of laminar flame speeds using propagating spherical flames, Combust. Theor. Model. 13 (2) (2009) 343364.

[22] A. Omari, L. Tartakovsky, Measurement of the laminar burning velocityusing the confined and unconfined spherical flame methods - A comparative analysis, Combust. Flame 168 (2016) 127-137.

[23] C.C.M. Luijten, E. Doosje, L.P.H. de Goey, Accurate analytical models for fractional pressure rise in constant volume combustion, Int. J. Therm. Sci. 48 (6) (2009) 1213-1222.

[24] E. Van den Bulck, Closed algebraic expressions for the adiabatic limit value of the explosion constant in closed volume combustion, J. Loss. Prev. Process Ind. 18 (1) (2005) 35-42.

[25] M. Jafargholi, G.K. Giannakopoulos, C.E. Frouzakis, K. Boulouchos, Laminar syngas-air premixed flames in a closed rectangular domain: DNS of flame propagation and flame/wall interactions, Combust. Flame 188 (2018) 453-468.

[26] Y. Yamamoto, T. Tachibana, Burning velocities of dimethyl ether (DME)-nitrous oxide $\left(\mathrm{N}_{2} \mathrm{O}\right)$ mixtures, Fuel 217 (2018) 160-165.

[27] F. Wu, C.K. Law, An experimental and mechanistic study on the laminar flame speed, Markstein length and flame chemistry of the butanol isomers, Combust. Flame 160 (12) (2013) 27442756.

[28] G. Rozenchan, D.L. Zhu, C.K. Law, S.D. Tse, Outward propagation, burning velocities, and chemical effects of methane flames up to $60 \mathrm{~atm}$, Proc. Combust. Inst 29 (2) (2002) 1461-1470.

[29] G. Wang, Y. Li, W. Yuan, Y. Wang, Z. Zhou, Y. Liu, J. Cai, Investigation on laminar flame propagation of $n$-butanol/air and $n$-butanol $/ \mathrm{O}_{2} / \mathrm{He}$ mixtures at pressures up to $20 \mathrm{~atm}$, Combust. Flame 191 (2018) 368-380.

[30] B. Galmiche, F. Halter, F. Foucher, Effects of high pressure, high temperature and dilution on laminar burning velocities and Markstein lengths of iso-octane/air mixtures, Combust. Flame 159 (11) (2012) 3286-3299.

[31] M. Goswami, R.J.M. Bastiaans, L.P.H. De Goey, A. Konnov, Experimental and modelling study of the effect of elevated pressure on ethane and propane flames, Fuel 166 (2016) 410-418.

[32] R.T.E. Hermanns, A. Konnov, R.J.M. Bastiaans, L.P.H. De Goey, K. Lucka, H. Köhne, Effects of temperature and composition on the laminar burning velocity of $\mathrm{CH}_{4}+\mathrm{H}_{2}+\mathrm{O}_{2}+\mathrm{N}_{2}$ flames, Fuel 89 (2010) 114-121.

[33] J. Natarajan, Y. Kochar, T. Liuwen, J. Seitzman, Pressure and preheat dependence of laminar flame speeds of $\mathrm{H}_{2} / \mathrm{CO} / \mathrm{CO}_{2} / \mathrm{O}_{2} / \mathrm{He}$ mixtures, Proc. Combust. Inst 32 (2009) 1261-1268.

[34] Y. Wu, B. Rossow, V. Modica, X. Yu, L. Wu, F. Grisch, Laminar flame speed of lignocellulosic biomass-derived oxygenates and blends of gasoline/oxygenates, Fuel 202 (2017) 572-582.

[35] M. Akram, S. Kumar, Experimental studies on dynamics of methane-air premixed flame in meso-scale diverging channels, Combust. Flame 158 (2011) 915-924.

[36] Z. Liu, M.J. Lee, N.I. Kim, Direct prediction of laminar burning velocity using an adapted annular stepwise diverging tube, Proc. Combust. Inst 34 (2013) 755-762.

[37] P. Versailles, G. Bourque, A. Durocher, J.M. Bergthorson, Measurements of the reactivity of premixed, stagnation, methane-Air flames at gas turbine relevant pressures, Proceedings of ASME Turbo Expo (2018).

[38] Z. Zhao, J.P. Conley, A. Kazakov, F.L. Dryer, Burning Velocities of Real Gasoline Fuel at $353 \mathrm{~K}$ and $500 \mathrm{~K}$, SAE Technical Paper 2003-01-3265 (2003).

[39] A.G. Gaydon, H.G. Wolfhard, Flames: Their Structure, Radiation and Temperature, London: Chapman and Hall Fourth Edition (1978).

[40] M. Slack, A. Grillo, High temperature rate coefficient measurements of CO+O chemiluminescence, Combust. Flame 59 (1985) 189-196.

[41] J.M. Samaniego, F.N. Egolfopoulos, C.T. Bowman, CO2* Chemiluminescence in Premixed Flames, Combust. Sci. Technol. 109 (1-6) (1995) 183-203.

[42] V. Nori, J. Seitzman, Evaluation of chemiluminescence as a combustion diagnostic under varying operating conditions, 46th AIAA Aerospace Sciences Meeting and Exhibit (2008). 
[43] D. Bradley, P.H. Gaskell, X.J. Gu, Burning velocities, markstein lengths, and flame quenching for spherical methane-air flames: A computational study, Combust. Flame 104 (1-2) (1996) 176198.

[44] F. Parsinejad, J.C. Keck, H. Metghalchi, On the location of flame edge in Shadowgraph pictures of spherical flames: a theoretical and experimental study, Exp. Fluids 43 (6) (2007) 887-894.

[45] R.J. Kee, F.M. Rupley, J.A. Miller, Chemkin-II: a Fortran chemical kinetics package for the analysis of gas phase chemical kinetics, Report No. SAND89-8009B, Sandia National Laboratories (1989).

[46] G.P. Smith, D.M. Golden, M. Frenklach, N.W. Moriarty, B. Eiteneer, M. Goldenberg, C.T. Bowman, R.K. Hanson, S. Song, W.C. Gardiner, V.V. Lissianski, Z. Qin, (http://www.me.berkeley.edu/gri mech/.

[47] M. Kopp, M. Brower, O. Mathieu, E. Petersen, F. Güthe, $\mathrm{CO}_{2}{ }^{*}$ chemiluminescence study at low and elevated pressures, Appl. Phys. B 107 (3) (2012) 529-538.

[48] A.E. Dahoe, Laminar burning velocities of hydrogen-air mixtures from closed vessel gas explosions, J. Loss. Prev. Process Ind. 18 (3) (2005) 152-166.

[49] Z. Chen, X. Qin, B. Xu, Y. Ju, F. Liu, Studies of radiation absorption on flame speed and flammability limit of $\mathrm{CO}_{2}$ diluted methane flames at elevated pressures, Proc. Combust. Inst 31 (2007) 2693-2700.

[50] E. Varea, V. Modica, A. Vandel, B. Renou, Measurement of laminar burning velocity and Markstein length relative to fresh gases using a new postprocessing procedure: Application to laminar spherical flames for methane, ethanol and isooctane/air mixtures, Combust. Flame 159 (2) (2012) 577-590.

[51] K. Eisazadeh-Far, A. Moghaddas, J. Al-Mulki, H. Metghalchi, Laminar burning speeds of ethanol/air/diluent mixtures, Proc. Combust. Inst 33 (1) (2011) 1021-1027. 